\title{
Development of a hybrid framework to characterize red lesions for early detection of diabetic retinopathy
}

\author{
Deepashree Devaraj, Prasanna Kumar S.C. \\ Department of Electronics and Instrumentation Engineering, RVCE, Bengaluru-59, India
}

\begin{tabular}{l}
\hline \hline Article Info \\
\hline Article history: \\
Received Jul 08, 2018 \\
Revised Nov 1, 2018 \\
Accepted Nov 28, 2018 \\
\hline
\end{tabular}

\section{Keywords:}

Hemorrhages

Local entropy Thresholding

Microaneurysms

Morphology

Quick propagation Neural

Network

\begin{abstract}
Diabetic retinopathy (DR) is one of the driving reasons for visual deficiency, affecting people globally. Currently, the ophthalmologists need to inspect enormous number of images with a specific end goal to perform mass screening of Diabetic retinopathy. In this paper, an efficient Computer aided system based on a Hybrid framework is proposed for the early diagnosis of DR by extracting the early DR lesions such as microaneurysms and hemorrhages. The development of such a screening system would decrease the workload of the ophthalmologists, as they now need to look at those retinal images that are analyzed by the system, as irregularities. The retinal images obtained from standard retinal databases and Hospitals are preprocessed followed by the detection and elimination of blood vessels, optic disk and exudates. Quick propagation Neural Network is used for training and testing of the retinal fundus images since it has the fastest execution time. Linear Classification and Multi class classification of retinal fundus images are performed for the classification and grading of retinal fundus images into normal and abnormal using Alyuda Neuro-Intelligence software. A patient database is created using MySQL to store the required details of the patient and a graphical user interface is developed for an efficient usage of the system. The execution time of the system is found to be 7-9 seconds and is tested on 270 retinal fundus images. The precision and accuracy of the algorithm is $92.5 \%$ and $93.9 \%$, respectively.
\end{abstract}

Copyright $@ 2019$ Institute of Advanced Engineering and Science. All rights reserved.

\section{Corresponding Author:}

Deepashree Devaraj,

Department of Electronics and Instrumentation Engineering,

RVCE, Bengaluru-59, India.

Email: deepashree@rvce.edu.in

\section{INTRODUCTION}

Diabetes essentially happens when the pancreas fails to secrete sufficient insulin for metabolism. It is a long term condition that causes very high glucose levels. Diabetic Retinopathy (DR) is a leading eye related disorder affected by diabetes. Since, it is asymptomatic in the initial stage; an efficient screening will prevent blindness. Various Image processing algorithms are used for the detection of Microaneurysms and Hemorrhages, which are the early clinical signs of DR. The detection of the early DR lesions and classification will help in combating blindness across the world.

It is highly important for diabetic patients to have regular eye check-ups. Current retinal examination systems, which are utilized for locating and reviewing diabetic retinopathy, incorporate Ophthalmoscopy (which may be indirect and direct), Fluorescein angiography, and Fundus photography. These methods of assessment and detection of DR are currently manual, expensive and requires trained ophthalmologists. There is significant need of ophthalmologists across the world, especially in the rural areas. A computer aided diagnosis (CAD) system would tackle the problem by increasing the mass screening of the early DR patients before consulting the ophthalmologists. This in turn reduces the time consumption and increases the efficiency of the diagnosis of the disease. 
The retinal features are macula, blood vessels, fovea and optic disc (OD). Any variations in these features may result in different retinal abnormalities. DR is of two types, namely Non proliferative DR (NPDR) and proliferative DR (PDR). Many features such as Microaneurysms (MAs), Hemorrhages (HMs), Exudates (hard and cottonwool spots) and Intraretinal Microvascular Abnormalities (IRMA) exists in NPDR. The very first sign related to early DR are microaneurysms (MAs) associated with the local expansions of the retinal capillaries and that result in hemorrhages (HA) when ruptured. In PDR, very small blood vessels develop from the retinal surface. The retinal fundus image with different features are as shown in Figure 1.

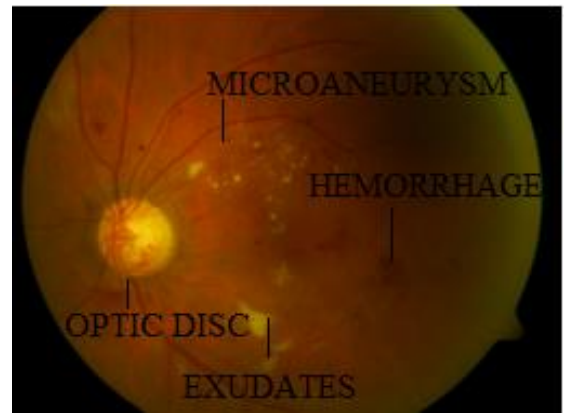

Figure 1. Abnormal Retinal Fundus Image

This paper proposes a CAD system for the early detection of DR to detect and characterise the red lesions based on a Hybrid technique clubbed with neural networks, a GUI that is user friendly and a patient database created using MySQL. The paper is organised in this fashion. A concise study of the research linked to the proposed system is presented in Section 2. A proficient early DR prediction system is presented in Section 3. The comprehensive results and discussions are given in Section 4. Conclusions are presented in Section 5 that sums up the explored work.

\section{RELATED WORK}

Most early screening programs use non-mydriatic digital fundus cameras to capture the retinal images. Retinal specialists then examine these images for the presence of lesions that are indicative of DR. However, the weak link is that the number of experts are considerably less and evaluating thousands of images is practically difficult. Thus, automatic detection via application of image processing techniques reduces the burden of the human experts.MA and HA are the earliest signs of DR. Blood vessels and OD needs to be eliminated for successful detection of early DR.

Blood vessels needs to be eliminated from the retinal images since it has similar intensity as that of microaneurysms. Annie et.al [1] proposed an algorithm for retinal blood vessel segmentation, which consisted of pre-processing stages, enhancement using fast discrete curvelet transform and multi structural element morphology to detect retinal blood vessels. Curvelet transform was then applied on the retinal fundus images. The limitations include the tedious calculation of the vascular parameters. Soares et.al [2] suggested a method for the vessel extraction using Gabor wavelet and Bayesian classifier. The accuracy was found to be $96 \%$. The method produced segmentation by categorizing every image pixel (as vessel or nonvessel), centered on pixel's features. Usman et.al [3] suggested a wavelet based method for the enhancement of the vessel along with the segmentation to obtain the region of interest. 2-D Gabor wavelet to augment the less visible vessels were used. Sensitivity was found to be around 94\%. Similar works were carried out by [4], [5] and [6]. Ben et.al [7] proposed a method based on morphological scale space. Line structuring element was rotated around the seed point to obtain the curvature of the vessels. It was performed on few images, which was one of the main limitation. Zhu et.al [8] proposed a supervised approach for identifying the retinal vessels based on extreme learning machine for pixel classification. Average accuracy was around $96 \%$. Hybrid classifiers could be used to improve the performance. Vasanthi et.al [9] carried out similar works. Akhavan et.al [10] suggested a method for the identification of vessels using fuzzy segmentation. The method showed consistent performance for normal as well as abnormal images.

Detection and removal of OD is also essential for the early detection of DR. Various methods have been proposed for the elimination of OD. Ana et al. [11] proposed a technique for the extraction of the vascular tree using a technique based on graph-cut approach. This information was used to locate the OD.

Development of a hybrid framework to characterize red lesions for early detection... (Deepashree Devaraj) 
The sensitivity was found to be less, which was a drawback. Akyol et. al. [12] proposed a technique for the identification of optic disk that included

image-processing, key-point extraction, texture-analysis, visual-dictionary, and classifier techniques. Accuracy of the method was found to be around 94\%. The technique worked well for images with noise and lesions. Murti et.al [13] suggested a method for the recognition of OD using least square fitting algorithm. Enhancement was done using histogram equalization and thresholding. Accuracy was found to be around 97.5\%. Less sensitivity and specificity were its major drawback. Rama Krishnan et al. [14] did similar works.

Microaneurysms and Haemorrhages were detected after the elimination of the Blood vessels and Optic disk. Different methods were adopted for the detection of these early DR lesions. Preeyaporn et al. [15] proposed a method that used a mix of HSV technique, identification of area along with eccentricity technique. A color bar was framed with the colors of arbitrarily identified MAs. The position of the MA was recognized if the dot in the image exists within the range of the target color. The accuracy was $93 \%$. It was found to have less sensitivity. Rukmini et al. [16] proposed another technique in light of fractal examination for the location of MAs. Fractal measurement was figured utilizing Box-Counting system. If the fractal dimension of a given image was lower than the threshold value, it was considered as normal or vice-versa. The sensitivity and the specificity were $89.5 \%$ and $82.1 \%$, which is the main limitation.

Akara Sopharak et al. [17] proposed a system in view of morphological operations for finding MA. After pre-processing, coarse segmentation was performed to distinguish the MA. Weka information mining programming and Naive Bayesian arrangement were utilized. The estimations of accuracy and precision were $83.34 \%$ and $99.99 \%$, respectively and sensitivity was found to be less. More improved results were achieved by [18]. Istvan et.al [19] proposed a system for the recognition of microaneurysm taking into account nearby rotational cross-segment profile analysis. Peak recognition was done on the acquired crosssectional profiles and afterwards factual measures were computed to get the MA. Naive Bayes (NB) classifier was utilized. The advantages were high sensitivity with low false positive rates. Marwan and Eswaran [20] proposed a system in which MAs and HAs were recognized for the early analysis of DR utilizing CLAHE and h-minima transform. Classifiers were not used, which was one of the major limitation. Niladri et al. [21] proposed a technique for the identification of MA, which comprised of division of the retinal image into four quadrants, followed by preprocessing in all the four quadrants, and later was concatenated. Execution time was one of the major limitations.

The survey of the literature works demonstrates that noteworthy works has been done in the field of identification of DR. Anyway, a framework with an automatic and early DR diagnosis system i.e., MA and HA as early DR signs is still not productive. The main goal of this work is development of a framework for the early recognition of DR. It expects to distinguish MAs, HAs from the normal images precisely, group and grade them utilizing the neural network system in conjunction with storage of the details in the patient database and a Graphical user interface(GUI) for the proficient and simple access of the framework. It focuses fundamentally on the development of a hybrid framework that can effectively locate the early DR features with an enhanced sensitivity, specificity, precision and accuracy.

\section{MATERIALS \& METHODS}

For effective detection of red lesions, the contrast between red lesions and the retinal background should be high and contrast between the retinal background and bright lesions should be low. This results in efficiently reducing the false positives during segmentation of candidate red lesions. Local entropy thresholding (LET) technique, takes the spatial distribution of gray levels into consideration and efficiently distinguishes enhanced dark lesions and the background as it can preserve the structural details of an image. Combining the best features of Local entropy thresholding and morphology (facilitates detection of smaller vessels), Hybrid method is proposed for the early detection of DR as shown in Figure 2. The CAD system for the early diagnosis of DR is divided into five phases: Acquisition of the Images, Pre-processing, Postprocessing which includes removal of features like Optic Disk, Blood Vessels, Exudates, Feature Extraction of MA and HA, Classification and storage of patient data in the database.

The retinal images are taken from many retinal fundus databases like DIARETDB0, DIARETDB1, and hospital databases of Prabha Eye Clinic and Narayana Nethralaya, Bengaluru. Then, green component of the image is taken since green color plane shows the best vessel contrast. Red Lesions appear brighter in the green plane. Optic Disk has features like constant size, high intensity and circular shape. Initially, the maximum value of the green component of the image and the median is calculated. Then, a circular mask is constructed at the center co-ordinates using (1). 


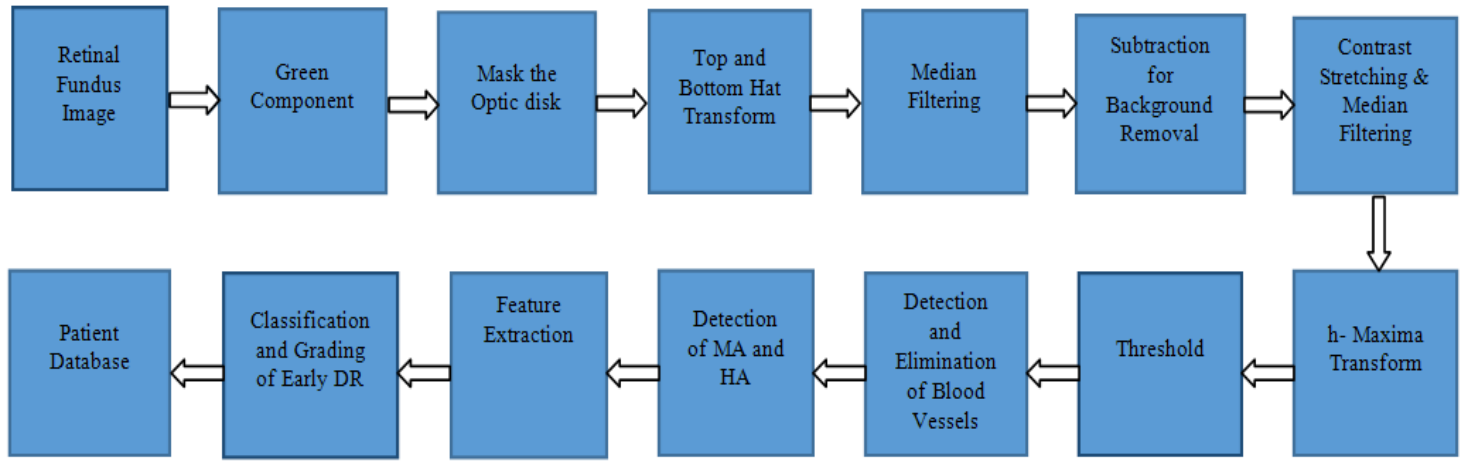

Figure 2. Block Diagram of the Hybrid method

$$
(a-p)^{2}+(b-q)^{2}=r^{2}
$$

Where, $\mathrm{r}$ is the radius and $(\mathrm{p}, \mathrm{q})$ are the center co-ordinates. The circular mask is then overlaid on the retinal image to get the OD masked image. Thus, Optic Disk is removed from the image. Background removal mainly aims at removing the background variations in the image. The foreground features are more prominent. Top and Bottom Hat Transform is applied to the OD masked image ( $f_{\text {od }}$ ). Top-hat operation is subtracting the result of performing a morphological opening on the OD masked image $\mathrm{f}_{\text {od }}$ from the OD masked image $\left(\mathrm{f}_{\mathrm{od}}\right)$ using structuring element $(\mathrm{SE})$ which is of type Ball as shown in (2).

$$
\mathrm{T}_{\text {hat }}\left(\mathrm{f}_{\mathrm{od}}\right)=\mathrm{f}_{\text {od }}-\left(\mathrm{f}_{\text {od }}{ }^{\circ} \mathrm{SE}\right)
$$

Where, SE is Ball structuring element of size 5 pixels and $\circ$ is the opening operation and $T_{\text {hat }}\left(f_{\text {od }}\right)$ is the image after performing Top Hat operation. It is then followed by Bottom Hat operation. Bottom Hat operation involves subtracting the result of Top hat operation from the closing operation performed on it using a Ball structuring element (SE) as shown in (3).

$$
\left.B_{\text {hat }}(x)=\left(T_{\text {hat }}\left(f_{\text {od }}\right) \cdot S E\right)-T_{\text {hat }}\left(f_{\text {od }}\right)\right)
$$

Where, $\mathrm{B}_{\text {hat }}(\mathrm{x})$ is the results of the Bottom Hat operation, $\mathrm{SE}$ is Ball structuring element of size 5 pixels and $\bullet$ is the Closing operation. It is then contrast enhanced using (4).

$$
I_{C E}=f_{\text {od }}+T_{\text {hat }}-B_{\text {hat }}
$$

Where, $\mathrm{I}_{\mathrm{CE}}$ is the Contrast Enhanced image, $\mathrm{T}_{\text {hat }}$ is the Top Hat Result and $\mathrm{B}_{\text {hat }}$ is the Bottom hat result. The contrast enhanced image is then median filtered $\left(\mathrm{I}_{\mathrm{med}}\right)$ and is then subtracted from contrast enhanced image for complete background removal process using (5).

$$
\mathrm{I}_{\mathrm{BN}}=\mathrm{I}_{\mathrm{med}}-\mathrm{I}_{\mathrm{CE}}
$$

Where $\mathrm{I}_{\mathrm{BN}}$ is the background removed image. The image is then contrast stretched to obtain the full dynamic range of the retinal image $\left(\mathrm{I}_{\mathrm{cs}}\right)$. It is then median filtered $\left(\mathrm{I}_{\mathrm{md}}\right)$ again using a structuring element. H-maxima transform is then applied to the median filtered image $\left(\mathrm{I}_{\mathrm{md}}\right)$ to suppress all the maxima in the intensity image $I_{m d}$ that is less than the threshold $h$, to obtain the image $f_{h}$. It is then thresholded with the value of 0.05 because features are not visible if the values are above 0.05 .

$$
\mathrm{f}_{\text {thx }}=\text { Threshold }\left(\mathrm{f}_{\mathrm{h}}, 0.05\right)
$$

Where, $f_{\text {thx }}$ is the thresholded image and $f_{h}$ is the $h$-maxima Transform. Thus, the resulting image consists of Blood vessels, MA and HA. Blood vessels are detected using a modified method of Local entropy Thresholding and Morphological method. Firstly, local entropy Thresholding is used for the detection of the blood vessels, where the Optimal Threshold is calculated as shown in the following equation.

$$
\mathrm{T}_{\mathrm{E}}=\left[\begin{array}{ll}
\max _{T=0}^{L-1} & \mathrm{H}_{\mathrm{T}}\left(\mathrm{T}_{\mathrm{h}}\right)
\end{array}\right]
$$


Where, $\mathrm{H}_{\mathrm{T}}\left(\mathrm{T}_{\mathrm{h}}\right)$ is the Second Order Entropy and $\mathrm{T}_{\mathrm{E}}$ is the optimal threshold based on which the vessels are thresholded from the image. Detection of blood vessels $\left(f_{b v 1}\right)$ is done using local entropy Thresholding method. Secondly, morphological detection of the blood vessels is done to enhance the detection of minor vessels. Morphological Blood Vessel detection involves the following steps. The green component of the image is extracted $\left(f_{g}\right)$. It is then inverted to obtain better enhancement.

$$
f_{i}=255-f_{g}
$$

Where, $f_{i}$ is the inverted image and $f_{g}$ is the green component image. After inversion of the image, canny edge detection is performed, which is a multistage algorithm to detect various edges.

$$
\mathrm{f}_{\mathrm{c}}=\operatorname{canny}\left(\mathrm{f}_{\mathrm{i}}\right)
$$

Where, $f_{c}$ indicates Canny detected image and $f_{i}$ is the inverted image. The image is dilated as well as eroded. The eroded image $\left(f_{e}\right)$ is subtracted from the dilated image $\left(f_{d}\right)$ to detect the border using the disk-shaped structuring element of radius 10 pixels.

$$
f_{b}=f_{d}-f_{e}
$$

Where, $\mathrm{f}_{\mathrm{b}}$ is the Border detected image, $\mathrm{f}_{\mathrm{d}}$ is the dilated image and $\mathrm{f}_{\mathrm{e}}$ is the eroded image. Contrast Limited Adaptive Histogram Equalization (CLAHE) is then applied on the green component image $\left(\mathrm{f}_{\mathrm{cl}}\right)$ ). A morphological opening operation was performed on the image using structuring element (SE) of ball shape as given by (11).

$$
\mathrm{f}_{\mathrm{o}}=\mathrm{f}_{\mathrm{cl}} \circ \mathrm{SE}
$$

Where, $f_{o}$ is the result of opening operation, $f_{\mathrm{cl}}$ is the concatenated CLAHE image and SE is the structuring element of disk shape. The opened image $\left(f_{o}\right)$ then subtracted from the CLAHE $\left(f_{c l}\right)$ performed image and then thresholded $\left(f_{t h}\right)$. It is then median filtered for preserving the edges. The obtained image is then subtracted from the Border detected image $\left(f_{b}\right)$. The resultant image contains the detected Blood Vessels $\left(f_{\text {bv2 }}\right)$. Then, the results of both the blood vessel detection methods are combined to obtain enhanced blood vessel

detection as given by (12).

$$
f_{\text {finalbv }}=f_{\text {bv1 }}+f_{\text {bv2 }}
$$

Where, $f_{\text {finalbv }}$ is the final detected blood vessel, $f_{\text {bvl }}$ is the blood vessel detected using local entropy thresholding and $\mathrm{f}_{\mathrm{bv} 2}$ is the blood vessel detected using morphology. The resultant blood vessels are again dilated using a disk SE. And finally it is subtracted from $\mathrm{f}_{\text {thx. }}$.

$$
\mathrm{f}_{\text {final }}=\mathrm{f}_{\text {thx }}-\mathrm{f}_{\text {finalbv }}
$$

Where, $\mathrm{f}_{\text {finalbv }}$ is the detected blood vessel, $\mathrm{f}_{\text {thx }}$ is the image containing MA, HA, blood vessels and $\mathrm{f}_{\text {final }}$ is the final MA, HA detected image. Thus, the image containing MA and HA are detected in the image. They are then separated based on the number of pixels into MA and HA. Hence, after the detection of MA and HA, features are extracted for classification.

\subsection{Feature Extraction}

Feature extraction helps in efficiently representing the interesting portions of an image as feature vector. Features like Area, Perimeter, Eccentricity $\left(\mathrm{E}_{\mathrm{cc}}\right)$, Major Axis Length (MAL), Minor Axis Length (MIL), Energy (E), Contrast (C) and Homogeneity (H) are extracted. Area is the number of white pixels existing in the region of interest in the binary image. Perimeter is the number of white pixels present in the boundary of the region of interest in the binary image.

Eccentricity is the measure of deviation of the conic section from being circular. It is given by,

$$
E_{c c}=\sqrt{\left(1-\frac{m^{2}}{n^{2}}\right)}
$$

Where, $\mathrm{m}$ - semi-major axis and n-semi-minor axis and e is the eccentricity. 
Contrast is the separation between the brightest and the darkest areas of the image. It is calculated as,

$$
\mathrm{C}=\sum_{i} \sum_{j} \mathrm{D}^{2}|\mathrm{i}, \mathrm{j}|
$$

Where, D|i,j| is the Normalized GLCM.

Energy is the sum of the elements that are squared in the matrix. It is calculated as,

$$
\mathrm{E}=\sum_{i} \sum_{j}(i-j)^{2} \mathrm{D}|\mathrm{i}, \mathrm{j}|
$$

Homogeneity measures the proximity of the element's distribution in the matrix. It is given by,

$$
\mathrm{H}=\sum_{\mathrm{i}} \sum_{\mathrm{j}} \frac{\mathrm{D}|\mathrm{i}, \mathrm{j}|}{1+|\mathrm{i}-\mathrm{j}|}
$$

Along with these features, total number of MAs and HAs are calculated.

\subsection{Classification}

Neural Network is used for Classification after the extraction of the features. Quick propagation Neural Network is used. The numbers of hidden Layers used are two and activation function used is Sigmoid Function. The method for Linear and Multi class classification of early Diabetic Retinopathy using Alyuda Neuro Intelligence Software are discussed below.

Firstly, the features extracted from the Training set data are stored in the Excel Sheets in Comma separated value (CSV) Format. One Excel Sheet contains the total number of Microaneurysms and Haemorrhages along with type of grading according to Table 1 as suggested by Dupas et al. [22]. Another Excel Sheet contains the six features extracted each for Microaneurysms and Haemorrhages. Secondly, after the creation of the Test data set using Excel sheet, Alyuda Neuro Intelligence Software is used. Thirdly, the test data file (Excel Sheet) containing the number of MAs and HAs along with the respective grading criteria specified in the last column, is loaded for Multi class classification of early DR i.e., Normal, Mild, Moderate and Severe. The Test data file containing 12 features of MA and HA is loaded for Linear classification seperately i.e., Normal and Abnormal Early DR Images.Finally, the data is analyzed and pre-processed. It is then followed by selecting the design, where the type of neural network is chosen as Quick Propagation Neural Network, and the number of Hidden Layers as two for training. It is then tested to see the classifier results. And the test results displayed on the GUI is fed for linear and Multi-class classification respectively for the classification results to be displayed.

Table 1. Rule Based Grading of Early Diabetic Retinopathy

\begin{tabular}{ccc}
\hline Early Diabetic Retinopathy Stadium & Number of Microaneurysms & Number of Haemorrhages \\
\hline Normal & 0 & 0 \\
Mild & $1 \leq$ MAs $\leq 5$ & 0 \\
Moderate & $5 \leq$ MAs $\leq 15$ & $0 \leq$ HAs $\leq 5$ \\
Serious & MAs $\geq 15$ & HAss $>5$ \\
\hline
\end{tabular}

\subsection{Patient Database Management System}

Patient Database management system is created using MySQL for storing the details of patients. It has records related to Patient ID, Name, Age, Weight, Gender, and Date, Patient History like Diabetes, Glaucoma, Cataract and the Count of Microaneurysms and Haemorrhages obtained by the hybrid method. A Graphical User Interface is created using MATLAB R2016a. It is built using push buttons, popup menus, panels, edit text boxes, static text boxes and axes. It consists of display options for the various stages of the algorithm for the detection of MA and HA along with the Execution Time, Classification using Neural Network and Patient Database Management System.

\section{RESULTS AND DISCUSSIONS}

The Retinal fundus image is obtained as shown in Figure 3(i), The Green component of the image is taken with the Optic disk masked image overlaid on it as shown in Figure 3(ii), The Top and Bottom Hat Transforms are applied on the image for background removal. Firstly, Top Hat is performed and then Bottom-Hat is performed as shown in Figure 3(iii). Background removal along with contrast enhancement is done for eliminating uneven variations as shown in Figure 3(iv).To increase the dynamic range of the retinal 
image, contrast stretching is done as shown in Figure 3(v) and it is then median filtered, thus preserving all the edges and reducing the salt and pepper noise as shown in Figure 3(vi). h-Maxima is then applied on the image to suppress the values less than a definite intensity as shown in Figure 3(vii). It is then thresholded as shown in Figure 3(viii). The blood Vessel is detected using Local Entropy thresholding and morphological method as shown in Figure 3(ix). After the detection of blood vessels by the hybrid method, it is subtracted from Figure 3(viii) to obtain HAs and MAs. Number of pixels present in the connected regions distinguishes HA and MA. Finally, HAs and MAs are detected as shown in Figures 3(x) and 3(xi).

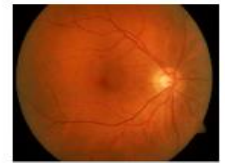

(i)

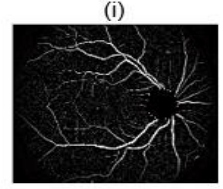

(v)

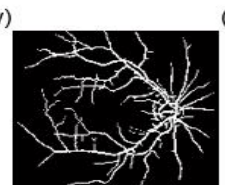

(ix)

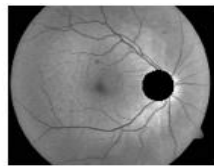

(ii)

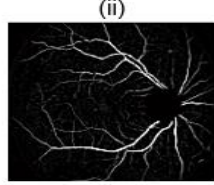

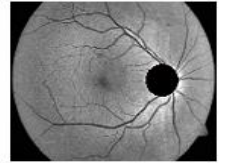

(iii)
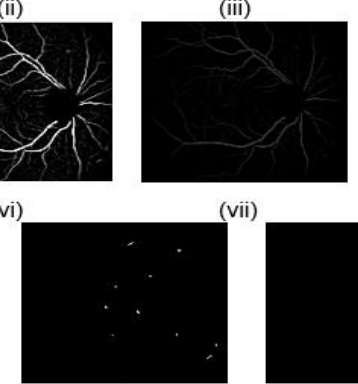

$(\mathrm{x})$

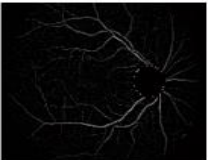

(iv)

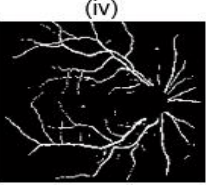

Figure 3. (i) Retinal Fundus Image (ii) Optic Disk mask overlaid on Green component image (iii) After Top and Bottom Hat Transform (iv) Background removal (v) Contrast Stretching (vi) Median Filtered (vii) h-Maxima Transform (viii)Thresholded (ix) Blood Vessel detection based on Hybrid method consisting of local entropy thresholding and morphological method (x) Haemorrhages (xi) Microaneurysms

\subsection{Results of Classification using Neural Network}

\subsubsection{Results of Linear Classification for Early Detection of Diabetic Retinopathy}

Various features like Area, Perimeter, Eccentricity, Major Axis, Minor axis, Contrast are extracted from Microaneurysms and Haemorrhages of the test data using Hybrid method for Early Detection of DR. The features extracted are entered in the Microsoft Excel sheet and the last column contains the classification of the training set as normal or abnormal DR for linear classification as shown in Figure 4. It is then loaded into Alyuda Neural Intelligence software where the neural network is analysed, pre-processed, designed and trained. The Quick propagation neural network is chosen, which uses sigmoidal activation function. The numbers of hidden layers used are two. It is then tested for 260 images, which are classified into Normal or Abnormal as shown in Figure 5.

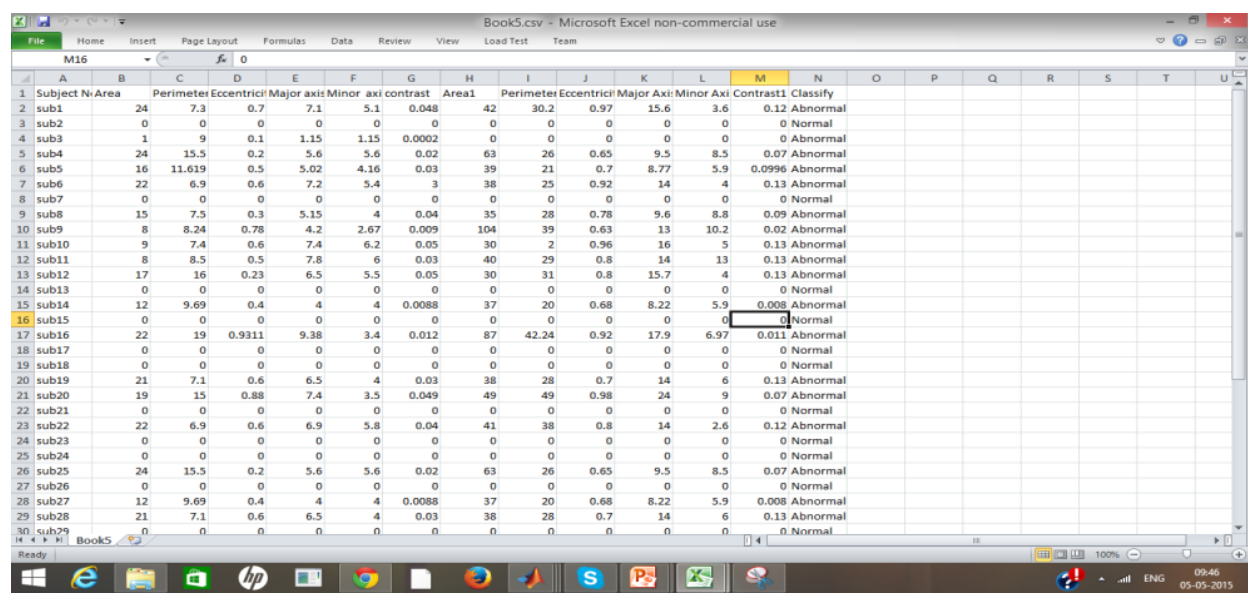

Figure 4. Features Extracted for Linear Classification 


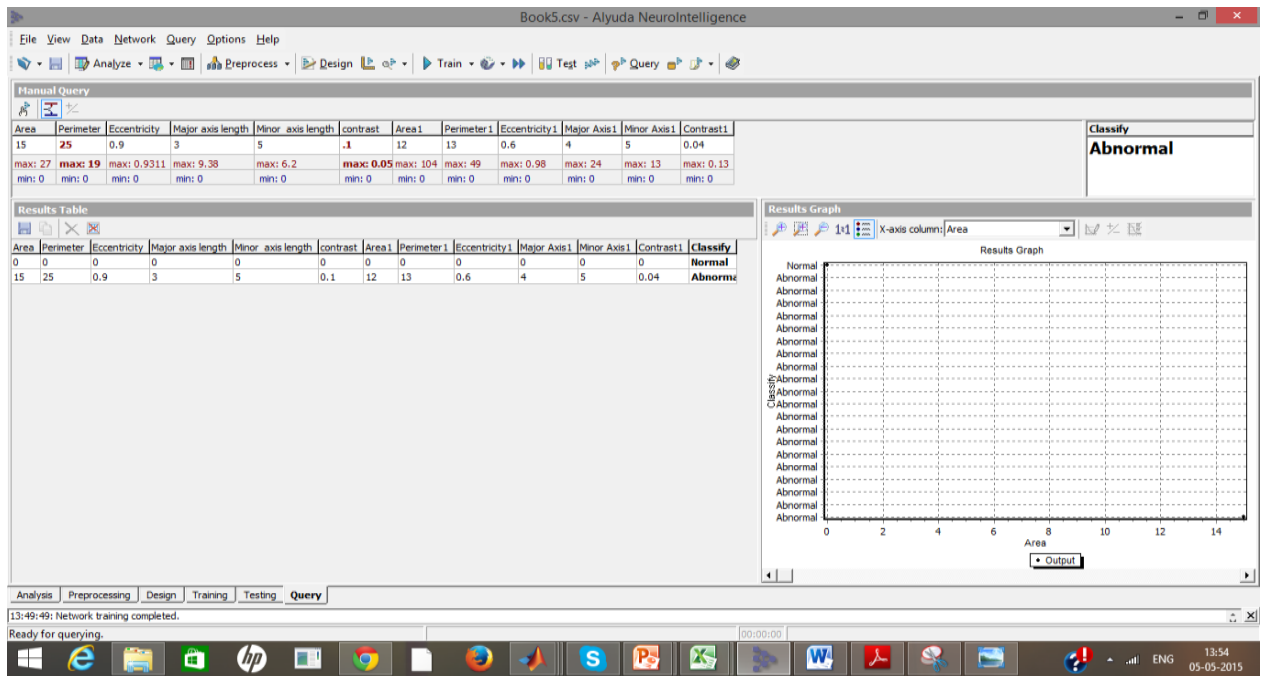

Figure 5. The Test Image classified as an Abnormal Image after entering the features

\subsubsection{Results of Multi Class Classification for Early Detection of Diabetic Retinopathy}

Number of Microaneurysms and Haemorrhages of the test data obtained from the Hybrid method are entered in the Microsoft Excel Sheet. They are used as the Test data as shown in Figure 6. It is then loaded into Alyuda Neural Intelligence software where, it is analysed, pre-processed, designed and trained. Once the system is trained, it is tested for 260 Images and graded into normal, mild, moderate and severe as shown in Figure 7.

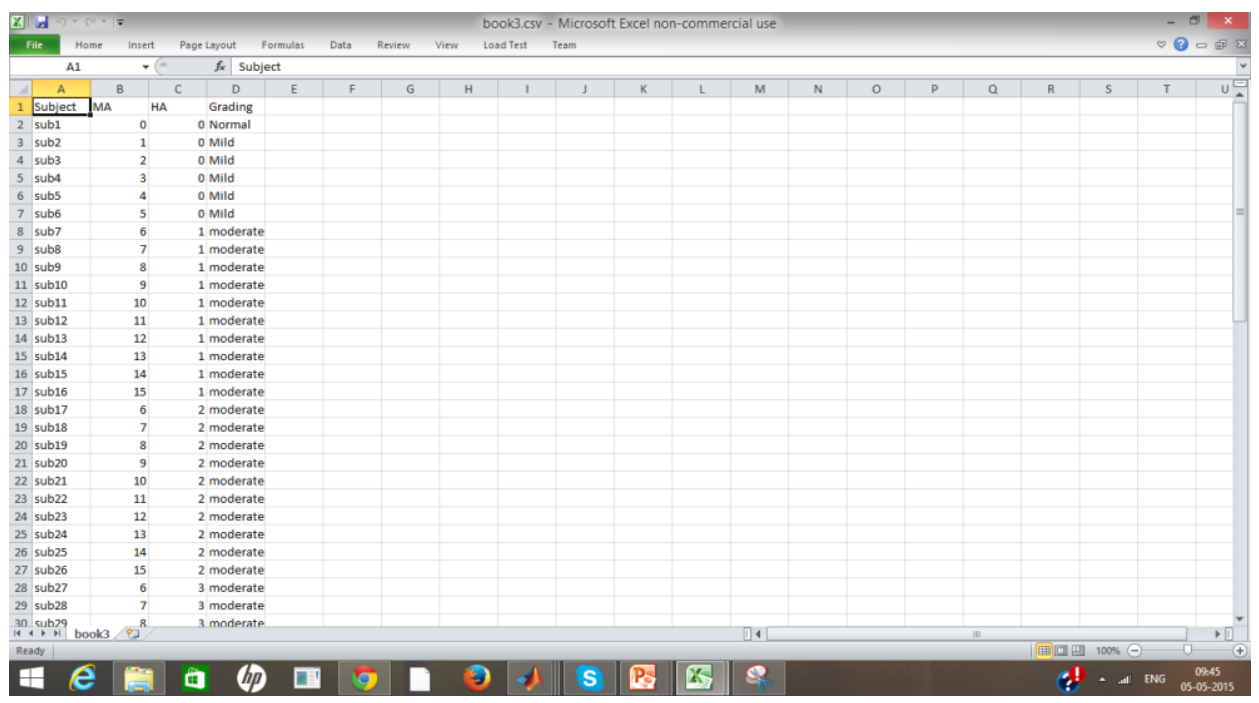

Figure 6. Features extracted for Multi class classification/Rule based grading 


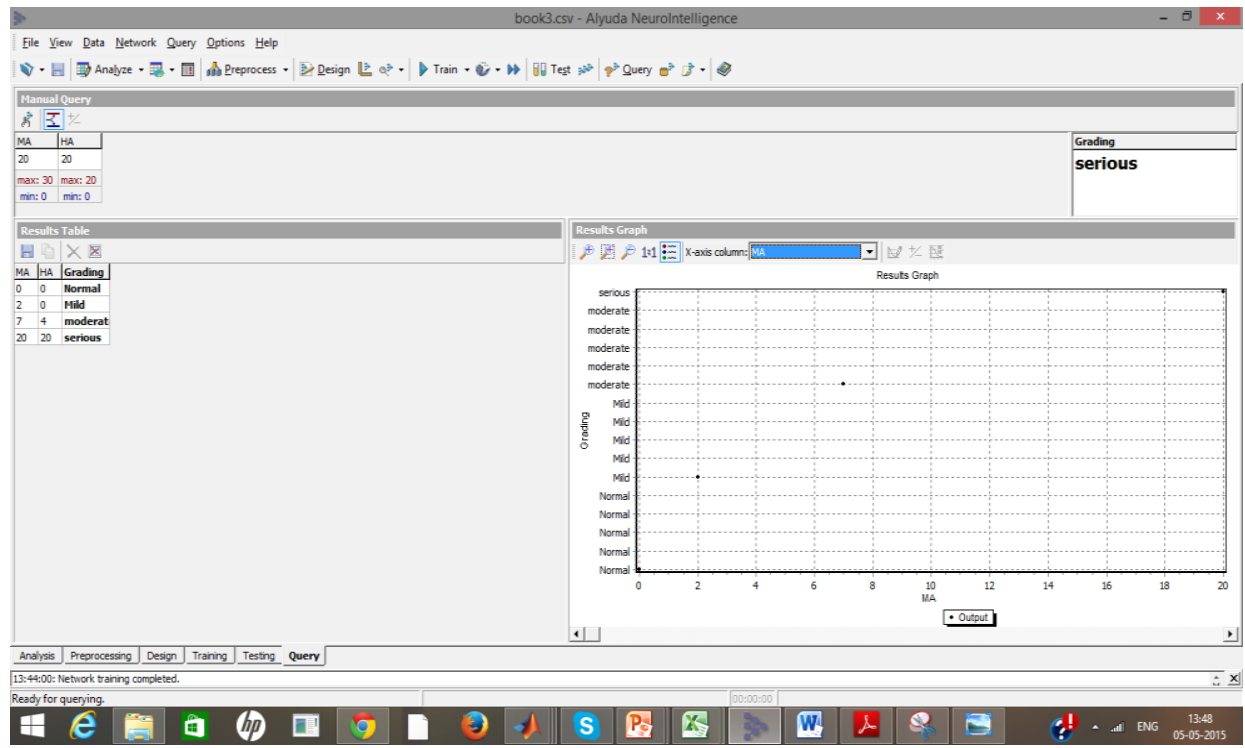

Figure 7. Results of Multi class classification/Rule based Grading

\subsection{Results of Patient Database Management System}

Patient Details like Patient ID, Name, Age, Weight, Gender, Date, Patient History like Diabetes, Glaucoma, Cataract and Count of Microaneurysms and Haemorrhages obtained by the Hybrid method are stored in the patient database created using MYSQL 5.5 as shown in Figure 8. Database created in MySQL was named as 'patient' and table created with all the records was named as 'Details'.

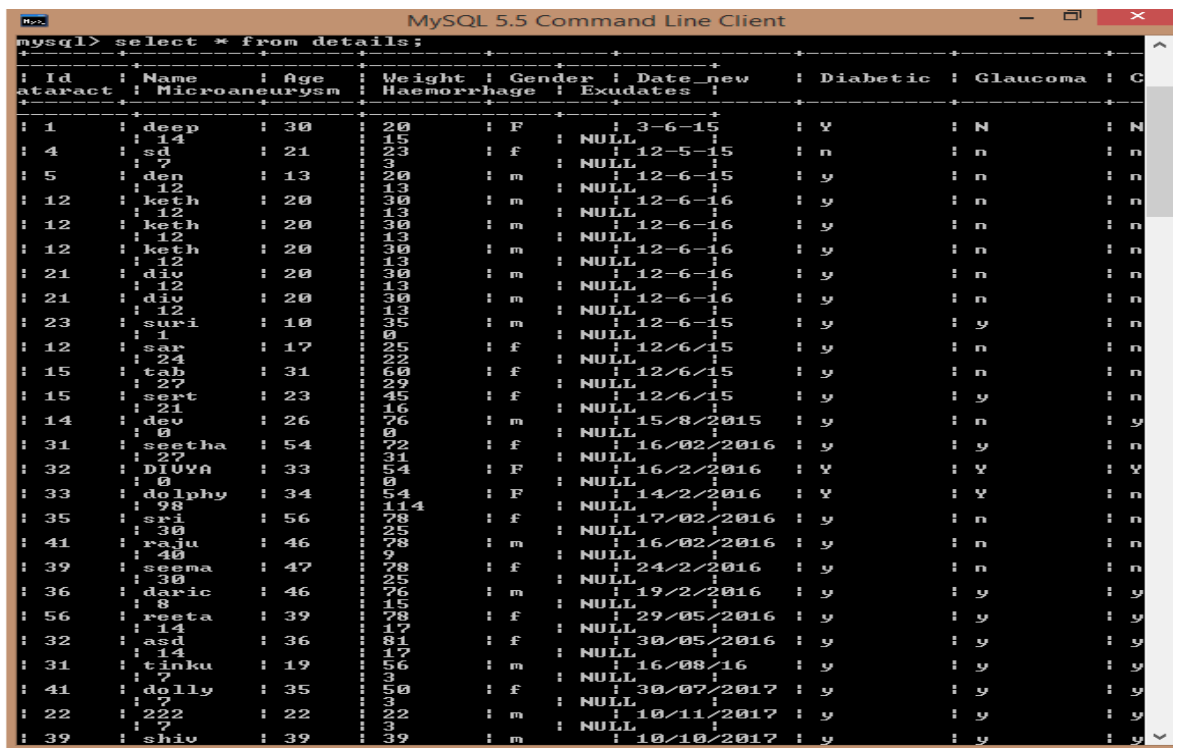

Figure 8. Early DR Patient Database created using MySQL

\subsection{Results of Graphical User Interface created for Early Detection of Diabetic Retinopathy}

A Graphical User Interface is created using MATLAB R2016a. It consists of display options for three algorithms for the detection of MA and HA along with the Execution Time, Classification using Neural Network and Patient Database Management System. The features extracted for the hybrid algorithm are displayed in the GUI. The patient details can be entered in the GUI, which is stored in the database. Figure 9 shows the GUI of the Early DR for a retinal fundus Image which is graded as moderate. It is observed that for the retinal image loaded, the number of MA's are 7 and the number of HA's are 3. 


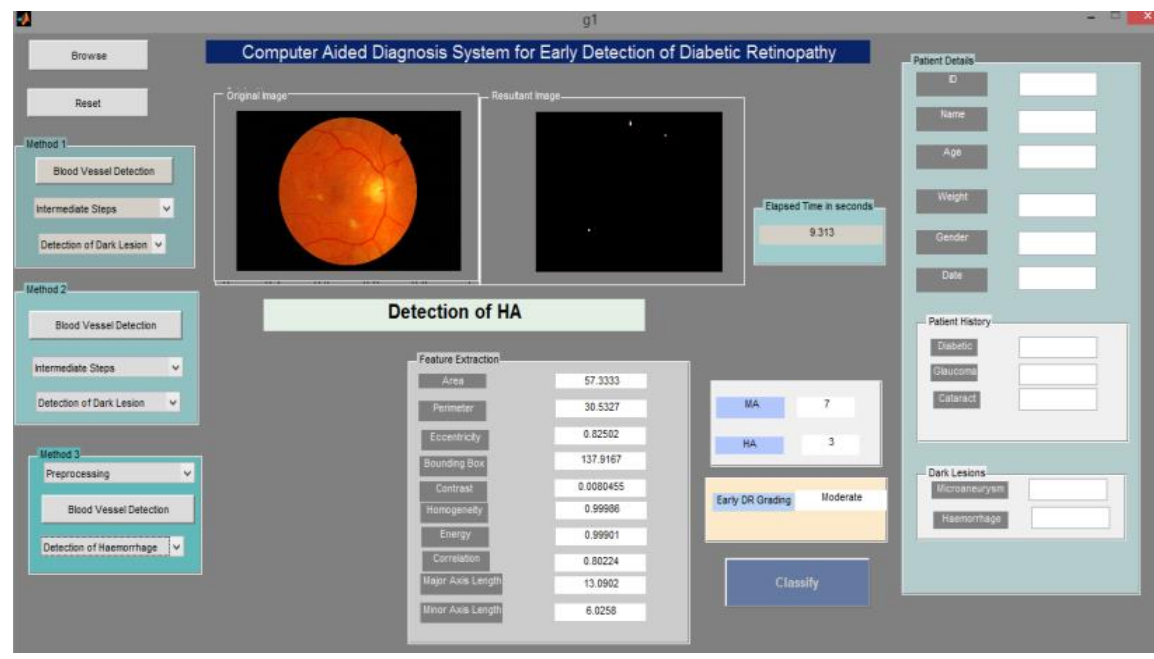

Figure 9. Graphical User Interface of the Retinal Fundus Image graded as moderate

Table 2 shows the performance analysis of the hybrid approach and the results for different databases using three different algorithms are presented in Figure 10.

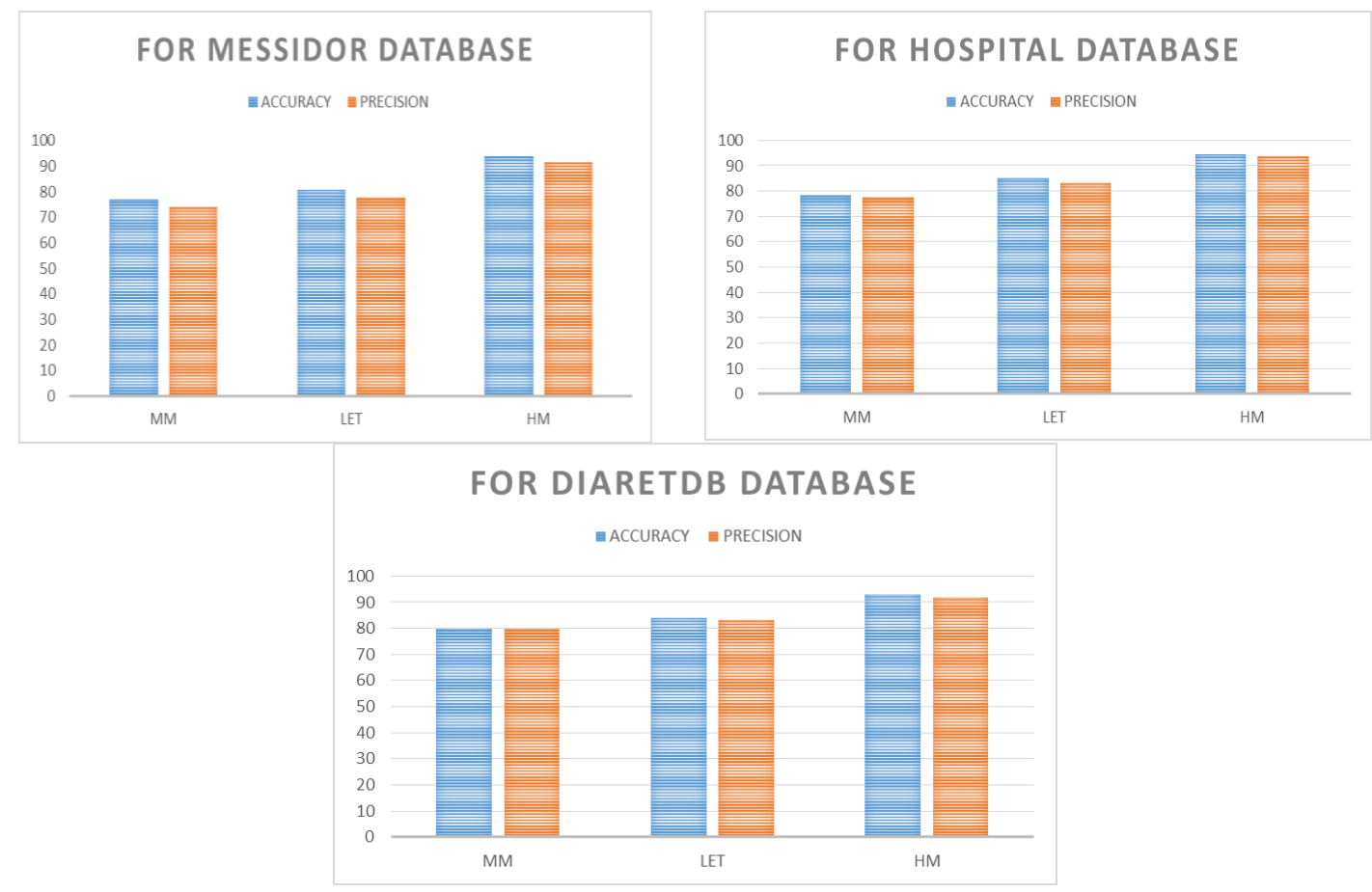

Figure 10. Performance Charts of the three different algorithms for different databases

The results of the work were compared with the ground-truths available for each image of the DIARETDB and MESSIDOR databases and validation was carried out for the hospital images by a Vitreoretinal specialist. The outcomes shows that the values of accuracy and precision have increased using the hybrid approach and for each fundus image the execution time is only 7-9s which is substantial. Table 3 illustrates the comparison of the 3 methodologies for the Detection of red lesions.In the Morphological method, some of the unique features identified are the division of an image into four quadrants before performing the pre-processing along with the masking of the optic disk using the centroid method. In the Entropy based method, some of the unique features identified are local entropy thresholding performed for detecting and eliminating the blood vessels, along with the active contour method for the detection and

Development of a hybrid framework to characterize red lesions for early detection... (Deepashree Devaraj) 
elimination of OD to detect the early DR lesions. In the Hybrid method, a unique combination of h-maxima transform, top-bottom hat transform, filling formed a part of pre-processing along with a modified combination of entropy thresholding and morphological method is used for the accurate detection of the blood vessels. Comparison of state-of-the art techniques for red lesion detection with the proposed method is presented in Table 4.

Table 2. Analysis of the Hybrid Approach

\begin{tabular}{ccccccc}
\hline Total no. of fundus images & DR & Normal & TP & FP & TN & FN \\
\hline 260 & 192 & 68 & 192 & 16 & 52 & 0 \\
\hline
\end{tabular}

Table 3. Comparison of the Three Methodologies for the Detection of Red lesions

\begin{tabular}{|c|c|c|c|}
\hline \multirow[t]{4}{*}{ Retinal Fundus Image } & Morphology Method(MM) & $\begin{array}{c}\text { Local Entropy } \\
\text { Thresholding(LET) }\end{array}$ & Hybrid Method(HM) \\
\hline & $\begin{array}{l}\text { Blood Vessel Detection is } \\
\text { not accurate in comparison } \\
\text { with the Ground Truth. } \\
\text { Minor Vessels are not } \\
\text { detected. }\end{array}$ & $\begin{array}{l}\text { Vessel Detection is } \\
\text { improved in comparison } \\
\text { with the Ground Truth. } \\
\text { Minor Vessels are detected. }\end{array}$ & $\begin{array}{l}\text { Accuracy of Vessel Detection } \\
\text { is far better when compared } \\
\text { with the Ground Truth(than } \\
\text { the } 2 \text { methods). All the blood } \\
\text { vessels are detected. }\end{array}$ \\
\hline & $\begin{array}{l}\text { Optic Disc detection is } \\
\text { accurate (Centroid Method). }\end{array}$ & $\begin{array}{l}\text { Optic Disc detection is } \\
\text { accurate (Centroid Method } \\
\text { and Active Contour } \\
\text { Method). }\end{array}$ & $\begin{array}{c}\text { Optic Disc Detection } \\
\text { (Centroid Method) is accurate. }\end{array}$ \\
\hline & $\begin{array}{l}\text { More number of False } \\
\text { positives for } \\
\text { Microaneurysms. }\end{array}$ & $\begin{array}{l}\text { Reduced number of False } \\
\text { positives for } \\
\text { Microaneurysm. }\end{array}$ & $\begin{array}{c}\text { Less number of False } \\
\text { positives for Microaneurysm. }\end{array}$ \\
\hline
\end{tabular}

Table 4. Comparison with Other State of the Art Techniques for Red Lesion Detection

\begin{tabular}{cccc}
\hline Authors & Execution Time & No. of images & Accuracy \\
\hline Kande et al[109] & $35 \mathrm{~s}$ & 89 & $93.23 \%$ \\
Syna Sreng et al[84] & $9.53 \mathrm{~s}$ & - & $90 \%$ \\
Hassan et al[54] & $1 \mathrm{~min}$ & 60 & $89 \%$ \\
Proposed method & $7-9 \mathrm{~s}$ & 260 & $93.9 \%$ \\
\hline
\end{tabular}

\section{CONCLUSIONS}

A characterization system for red lesions to facilitate early DR detection was developed. 260 retinal images obtained from different databases and hospitals were considered for testing. The method achieved an accuracy and precision of $93.9 \%$ and $92.5 \%$, respectively. The execution time was found to be 7-9 seconds. The automated system was developed using MATLAB R2016a and Alyuda Neuro Intelligence Tool was 
used to classify the retinal fundus images into normal and abnormal and grading of the retinal images based on the presence of the MA and HA as normal, mild, moderate and severe. A patient database management system created using MySQL helped in storing all the patient details for future reference and a user friendly Graphical User Interface was developed for the Early Detection of Diabetic Retinopathy.

\section{REFERENCES}

[1] Annie Edel Quinn E, Gokula KK. Retinal Blood Vessel Segmentation using Curvelet Transform and Morphological Reconstruction.Proceedings of International Conference on Emerging Trends in Computing, Communication and Nanotechnology. IEEE.2013; 570-575.

[2] Soares JVB, Leandro JJG, Cesar RM, Jelinek HF,Cree MJ. Retinal vessel segmentation using the 2-D Gabor wavelet and supervised classification. IEEE Transactions on Medical Imaging.2006; 25(9): 1214-1232.

[3] Usman AM, Aasia, K. Retinal Images: Blood Vessel Segmentation by Threshold Probing. Proceedings of Symposium on Industrial Electronics and Applications. Penang. Malaysia. 2010; 493-497.

[4] Usman AM, Ali A, Farrukh AS, Shoab AK. Blood Vessel Enhancement and Segmentation Using Wavelet Transform. Proceedings of the International Conference on Digital image processing. Washington. USA. 2009; $34-$ 38.

[5] Usman AM, Anam T, Sarwat N, Shoab AK. Gabor Wavelet Based Vessel Segmentation in Retinal Images. Proceedings of Symposium on Computational Intelligence for Image processing. Nashville. USA. 2009; 116-119.

[6] Usman Akram Retinal Image Preprocessing: Background and Noise Segmentation.Indonesian Journal of Electrical Engineering and Computer Science.2012; 10(3): 537-544.

[7] Ben KJ, Roland TC. Morphological Scale Space for 2D Shape Smoothing. Computer Vision and Image Understanding.1998; 70(2): 121-141.

[8] Zhu C, Zou B, Zhao R, Cui J, Duan X, Chen Z, Liang Y.Retinal vessel segmentation in colour fundus images using Extreme Learning Machine. Computerized Medical Imaging and Graphics. 2017; 55:68-77.

[9] Vasanthi Shanmugam, RSD Wahida Banu. Retinal Blood Vessel Segmentation using an Extreme Learning Machine Approach, Bonfring International Journal of Man Machine Interface. 1 Special Issue: 2011; 15-21.

[10] Razieh Akhavan, Karim Faez. A Novel Retinal Blood Vessel Segmentation Algorithm using Fuzzy segmentation. International Journal of Electrical and Computer Engineering.2014:4(4): 561-572.

[11] Ana SG, Djibril K, Yongmin L, Xiaohui L. Segmentation of the Blood Vessels and Optic Disk in Retinal Images IEEE Journal of Biomedical and Health Informatics.2014;18(6):1874-1886.

[12] Kemal Akyol, BahaŞen, ŞafakBayır. Automatic Detection of Optic Disc in Retinal Image by Using Keypoint Detection. Texture Analysis, and Visual Dictionary Techniques. Computational and Mathematical Methods in Medicine. 2016; 6814791.

[13] Murthi A, Madheswaran M, Enhancement of Optic Cup to Disc Ratio Detection in Glaucoma Diagnosis. Proceedings of International Conference on Computer Communication and Informatics. Coimbatore, India, IEEE, 2012; 1-5.

[14] Rama Krishnan MM, Rajendra AU, Chua KC, Lim CM, Milind MM, Augustinus L. Application of Intuitionistic Fuzzy Histon Segmentation for the Automated Detection of Optic Disc in Digital Fundus Images. Proceedings of International Conference on Biomedical and Health Informatics, Hong Kong, China, 2012; 444-447.

[15] Preeyaporn Y, Noppadol M, Ronakorn PMD, Ruttikorn V. Automatic Microaneurysms Detection through Retinal Color Image Analysis. Proceedings of International Conference on Information Technology and Electrical Engineering. Yogyakarta. Indonesia. 2013; 36-40.

[16] Rukhmini R, Srinivasan A, Partha B. Detection of Retinal Microaneurysms using Fractal Analysis and Feature Extraction Technique. Proceedings of International Conference on Communication and Signal Processing. Melmaruvathur. India. 2013; 469-474.

[17] Akara S, Bunyarit U, Sarah B. Simple hybrid method for fine microaneurysm detection from non-dilated diabetic retinopathy retinal images. Computerized Medical Imaging and Graphics. 2013; 37(5-6):394-402.

[18] Jeyapriya J, K S Umadevi, Jagadeesh R Kannan.Automated Detection of Microaneurysms using Probabilistic Cascaded Neural Network. Indonesian Journal of Electrical Engineering and Computer Science. 2018; 11(3):10831093.

[19] Istvan L, Andras H. Retinal Microaneurysm Detection through Local Rotating Cross-Section Profile Analysis. IEEE Transactions on Medical Imaging. 2013; 32(2):400-407.

[20] Marwan DS, Eswaran C. An automated decision-support system for non-proliferative diabetic retinopathy disease based on MAs and Has Detection. Computer Methods and programs in Biomedicine. 2012; 108(1): 186-196.

[21] Niladri SD, Himadri SD, Mallika D, Saurajeet M. An Effective Approach: Image Quality Enhancement for Microaneurysms Detection of Non-Dilated Retinal Fundus Image. Proceedings of First International Conference on Computational Intelligence: Modelling Techniques and Applications. Kalyani. India, Procedia Technology.2013; 10:731-737.

[22] Dupas, B, Walter T, Erginay A. Evaluation of automated fundus photograph analysis algorithms for detecting microaneurysms, haemorrhages and exudates, and of a computer-assisted diagnostic system for grading diabetic retinopathy. Diabetes \& Metabolism.2010; 36(3):213-220. 